\title{
Underperformance Fees and Manager's Portfolio Risk Taking
}

\author{
Gabriele Stabile ${ }^{1}$ \\ ${ }^{1}$ Dipartimento di Metodi e Modelli per l'Economia, il Territorio e la Finanza, Sapienza-Università di Roma, Italy \\ Correspondance: Gabriele Stabile, Dipartimento di Metodi e Modelli per l'Economia, il Territorio e la Finanza, \\ Sapienza-Università di Roma, via del Castro Laurenziano 9, 00161 Roma, Italy. E-mail: \\ gabriele.stabile@uniroma1.it
}

Received: December 5, 2014

Accepted: December 23, 2014

Online Published: December 29, 2014

doi:10.5430/ijfr.v6n1p79

URL: http://dx.doi.org/10.5430/ijfr.v6n1p79

\begin{abstract}
This paper investigates how a manager's compensation contract where good performance are rewarded and poor performance are penalized impacts on the managerial risk taking propensity. The results of the model indicate that the presence of underperformance penalty has a strong impact on the manager's investment strategies. As the asset value goes to zero, the optimal proportional portfolio goes to infinity. On the other hand, as the asset value goes to infinity, the optimal proportional portfolio converges to the Merton constant, that is the portfolio the manager chooses if he were trading his own account. In some situations, the manager's optimal portfolio is below the Merton constant. If the asset value is somewhat below the overperformance region, the manager chooses trading strategies more risky than the Merton constant. Thus, in order to assure that his incentive option will finish in-the-money, the manager increases the investment volatility, but not in the indiscriminate manner as he does in case of absence of underperformance penalty.
\end{abstract}

Keywords: optimal risk taking, managerial control, optimal portfolio choice

\section{Introduction}

This paper analyzes the implications of performance fee incentives allowing for both over- and under-performance on manager's portfolio risk taking. The hurdle rates from which over- and under-performance are measured may be different. In particular we assume that under-performance is rewarded if the managed assets value exceeds the value of an external benchmark, whereas under-performance fee is applied if the asset value goes below a predetermined percentage of the benchmark.

These incentive scheme considered includes the symmetric performance fee, where over- and under-performance are measured in respect to the same threshold, and the bonus performance fee where no penalty is applied in case of under-performance.

On behalf of the fund's owner, the manager selects a dynamic trading strategy, and for this the manager is compensated at a prescribed terminal time. We focus on the effect of incentives and fee structure on fund behaviour and on the risk level the manager will target. In the literature both absolute and relative fee structures are considered. In the former structure fees are related only to the asset value, whereas in the latter fees depend also on relative performance measured against a predetermined benchmark. Carpenter (2000) examines the manager's trading behavior in case the manager is compensated with a base fee plus a bonus if the asset value ends up above a stochastic benchmark. She finds that in some situations the manager chooses a lower asset volatility than he would if he were investing on his own. Hodder and Jackwerth (2007) investigate incentive effects of a typical hedge fund contract for a manager with power utility. They consider the possibility of liquidation if the fund performs poorly. Gupta and Skallsjo (2007) adopt a compensation scheme where over- and under-performance are measured in respect of two distinct hurdle rates. They study the optimal activeness policy of a hedge fund manager taking explicitly into account the manager's level of skill. Cuoco and Kaniel (2011) consider a compensation contract that includes penalties for underperforming the chosen benchmark that are symmetric to the bonuses for exceeding it. Their analysis is focused on the impact of delegated portfolio management on asset prices within a dynamic general equilibrium setting. Buraschi, Kosowski and Sritrakul (2014) consider a hedge fund manager's compensation scheme composed by a proportional fee, plus a bonus if the fund's value is above the high-water mark, minus a deadweight loss due to the deleveraging when the fund's value falls below another hurdle rate. 
In this paper we essentially adopt the compensation contract proposed in Gupta and Skallsjo (2007). The manager has power utility displaying constant relative risk aversion (CRRA). The manager aims to maximize the expected utility of his compensation at the terminal time. The optimal dynamic trading strategies are analytically derived and the results are illustrated by means of numerical examples. As the asset value goes to zero, the optimal proportional portfolio goes to infinity. On the other hand, as the asset value goes to infinity, the optimal proportional portfolio converges to the Merton constant, that is the portfolio the manager chooses if he were trading his own account. In some situations, the manager's optimal portfolio is below the Merton constant. If the asset value is somewhat below the overperformance region, the manager chooses trading strategies more risky than the Merton constant. Thus, in order to increase the probability to finish with an asset value at terminal time in the overperformance region, the manager increases the investment volatility, but not in the indiscriminate manner as he does in case of absence of underperformance penalty.

The rest of the paper is organized as follows. In Section 2 we setup the manager's optimal investment problem. In Section 3 we derive the manager's optimal payoff and the optimal trading strategy. In Section 4 the results are illustrated by means of numerical examples. Finally, Section 5 concludes.

\section{The Model}

We analyze our problem on a complete probability space $(\Omega, \mathcal{F}, P)$ with a filtration $\{\mathcal{F}(t)\}$ satisfying the usual condition and carrying a standard one-dimensional $(\mathcal{F}(t))$ - brownian motion $W(t)$. We consider a delegated portfolio management problem on a finite time interval $[0, T]$. At time $t=0$ the manager is endowed with a wealth equal to $x$. He has the possibility to invest in a frictionless financial market including a riskless bond $A$ and a risky stock $S$. The per unit prices of the financial products evolve according to

$$
\left\{\begin{array}{l}
d A_{t}=r A_{t} d t \\
d S_{t}=S_{t}\left(\mu d t+\sigma d W_{t}\right),
\end{array}\right.
$$

where $r, \mu$ and $\sigma$ are positive real constants. Let $\pi(\cdot)$ be the amount of wealth invested in stock at time $t$ (the riskiness of the project). The value of asset portfolio evolves in time according to the following stochastic differential equation

$$
\left\{\begin{array}{l}
d X_{t}=\left[r x_{t}+\pi_{t}(\mu-r)\right] d t+\sigma \pi_{t} d W_{t} \\
X_{0}=x,
\end{array}\right.
$$

At terminal time $T$ the manager receives the payment.

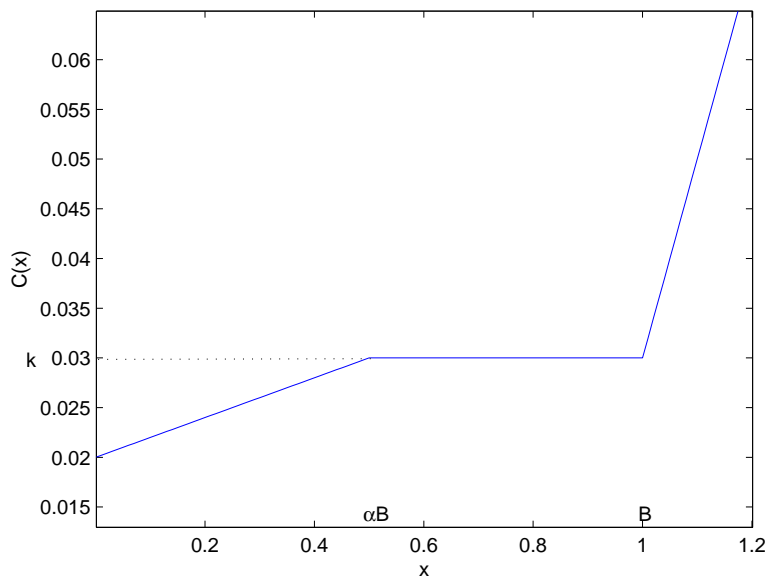

Figure 1. Compensation contract

The compensation contract (see Figure 1) has the following form

$$
C(x)=k+\theta_{H}(x-B)^{+}-\theta_{L}(x-\alpha B)^{-},
$$

where $k>0$ is the constant management fee, $\theta_{H}>0$ and $\theta_{L} \geq 0$ are respectively the overperformance and underperformance coefficients with $\theta_{H} \geq \theta_{L}, B>0$ is a given benchmark that in our setting is assumed to be a constant prespecified at the beginning of the contract, $\alpha \in(0,1]$ is the penalization boundary coefficient (Note 1 ). Manager's compensation is composed by a base fee $k$ plus a bonus if the value of asset portfolio exceeds the 
benchmark $B$. On the other hand, if the value of asset portfolio is below $\alpha B$, the manager incurs in a underperformance penalty. Notice that $C$ is increasing in wealth and decreasing in the benchmark.

The manager is guided by a constant relative risk aversion preferences

$$
U(x)=\frac{x^{1-\gamma}}{1-\gamma}
$$

with $\gamma>0$ and $\gamma \neq 1$.

The manager chooses a trading strategy $\pi$ in order to maximize his compensation at the terminal time

$$
\max _{\pi} E\left[U\left(C\left(X_{T}\right)\right)\right]
$$

\section{The Analysis}

Problem (4) is analyzed by using the so called martingale method to portfolio optimization, introduced in Cox and Huang (1989), Karatzas and Lehoczky and Shreve (1987) and Pliska (1986). The main idea is to decompose the dynamic optimization problem of choosing an optimal investment strategy $\pi$ into a static optimization problem (determination of the optimal terminal wealth) and a representation problem (find a portfolio strategy that leads to this optimal terminal wealth). Define

$$
u(x)=U(C(x))= \begin{cases}U\left(\theta_{L} x+k-\theta_{L} \alpha B\right) & \text { if } x \in[0, \alpha B] \\ U(k) & \text { if } x \in(\alpha B, B) \\ U\left(\theta_{H} x+k-\theta_{H} B\right) & \text { if } x \in[B,+\infty)\end{cases}
$$

for all $x \in[0, \infty)$. The static optimization problem is

$$
\max _{x_{T}} E\left[u\left(X_{T}\right)\right]
$$

subject to $\mathbf{E}\left[\xi_{T} X_{T}\right] \leq x$ and $X_{T} \geq 0$, where

$$
\xi_{t}=\exp \left[-\int_{0}^{t}\left(r_{s}+\frac{\theta_{s}^{2}}{2}\right) d s-\int_{0}^{t} \theta_{s} d W_{s}\right]
$$

is the state price density and $\theta=\frac{\mu-r}{\sigma}$ is the relative risk premium. Once we have solved the static problem, say $X_{T}^{*}$ the optimal payoff at time $T$, we will analyze the representation problem, i.e. find the portfolio investment strategy $\pi^{*}$ such that $X_{T}=X_{T}^{*}$, where $X_{T}$ is the solution of (1) with portfolio $\pi^{*}$.

Unless the case when $\alpha=1$ and $\theta_{L}=\theta_{H}$, the objective function $u(\cdot)$ is not globally concave. Moreover, $u$ is not differentiable at $x=\alpha B$ and $x=B$. Then we apply the concavification technique used in Carpenter (2000), that is we look for the smallest concave function that dominates $u(\cdot)$, we solve the problem with the concavified objective function by using standard methods and then we prove that the optimum never takes on values where the two functions disagree.

Through the paper we assume that $C(0)=k-\theta_{L} \alpha B \geq 0$ a.s. in order to guarantee that the manager receives non negative compensation (Note 2). In the sequel we will not analyze the cases when $\theta_{L}=0$ because in this case the manager's problem becomes the same analyzed in Carpenter (2000), or the case when $\alpha=1$ and $\theta_{L}=\theta_{H}$ because in this case the objective function $u$ is globally concave. 
(A)

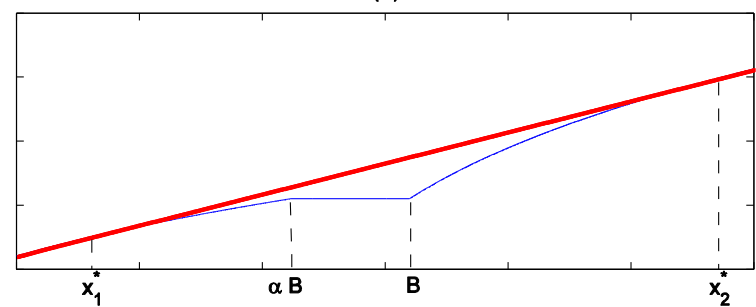

(B)

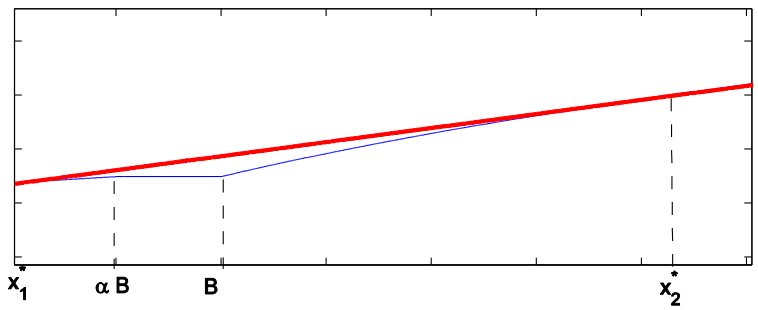

(C)

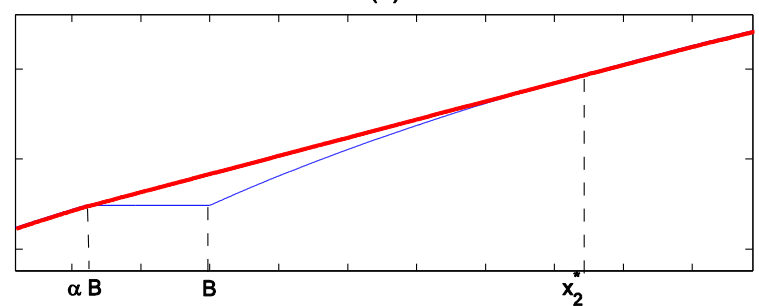

Figure 2. Concavification

Next lemma clarify the construction of the concavified objective function.

Lemma 3.1. Assume $\theta_{L}>0$. If $\theta_{L}=\theta_{H}$ and $\alpha<1$ fix $x_{1}^{*}=\alpha B$ and let $x_{2}^{*}>0$ be the unique solution of $\frac{u\left(x_{2}^{*}\right)-u(\alpha B)}{x_{2}^{*}-\alpha B}=u^{\prime}\left(x_{2}^{*}\right)$.

If $\theta_{L}<\theta_{H}$, then there exist $x_{1}$ and $x_{2}$, with $x_{1}<x_{2}$ and $x_{2}>B$, such that

$$
\left\{\begin{array}{l}
\frac{u\left(x_{2}\right)-u\left(x_{1}\right)}{x_{2}-x_{1}}=u^{\prime}\left(x_{2}\right) \\
u^{\prime}\left(x_{1}\right)=u^{\prime}\left(x_{2}\right)
\end{array}\right.
$$

In case $x_{1} \in(0, \alpha B)$, set $x_{1}^{*}=x_{1}$ and $x_{2}^{*}=x_{2}$. Otherwise, if $x_{1}<0$ set $x_{1}^{*}=0$, whereas if $x_{1}>\alpha B$ set $x_{1}^{*}=\alpha B$. In both cases, let $x_{2}^{*}>0$ be the unique solution of $\frac{u\left(x_{2}^{*}\right)-u\left(x_{1}^{*}\right)}{x_{2}^{*}-x_{1}^{*}}=u^{\prime}\left(x_{2}^{*}\right)$.

Then

$$
v(x)= \begin{cases}u(x) & \text { if } x \in\left[0, x_{1}^{*}\right) \cup\left(x_{2}^{*},+\infty\right) \\ u\left(x_{1}^{*}\right)+u^{\prime}\left(x_{2}^{*}\right)\left(x-x_{1}^{*}\right) & \text { otherwise, }\end{cases}
$$

is the smallest concave function satisfying $v(x) \geq u(x) \forall x \in[0, \infty)$.

The construction of the concavified objective function is discussed in Appendix A and it is illustrated in Figure 2.

Proposition 3.2. Assume $\theta_{L}>0$ and let $x_{1}^{*}$ and $x_{2}^{*}$ be as in Lemma 3.1.

The value of the asset portfolio and the optimal dynamic trading strategies are given by 
A. If $0<x_{1}^{*}<\alpha B$ then

$$
\begin{aligned}
& X_{t}^{*}=e^{-r(T-t)}\left[\left(x_{2}^{*}-B+\frac{k}{\theta_{H}}\right)\left(N\left(-d_{2, t}\right)+\left(\frac{\theta_{L}}{\theta_{H}}\right)^{\frac{1-\gamma}{\gamma}} N\left(d_{2, t}\right)\right) \frac{N^{\prime}\left(d_{1, t}\right)}{N^{\prime}\left(d_{2, t}\right)}\right] \\
& -e^{-r(T-t)}\left(\frac{k}{\theta_{L}}-\alpha B\right)\left(N\left(d_{1, t}\right)-N\left(d_{3, t}\right)+N\left(d_{4, t}\right) \frac{N^{\prime}\left(d_{3, t}\right)}{N^{\prime}\left(d_{4, t}\right)}\right) \\
& -e^{-r(T-t)}\left(\frac{k}{\theta_{H}}-B\right) N\left(-d_{1, t}\right)
\end{aligned}
$$

and

$$
\begin{aligned}
& \pi_{t}^{*}=\frac{\mu-r}{\sigma^{2}}\left\{\frac{X_{t}^{*}}{\gamma}+\frac{e^{-r(T-t)}}{\theta \sqrt{T-t}} N^{\prime}\left(d_{1, t}\right)\left(x_{2}^{*}+\frac{k}{\theta_{L}}-\alpha B\right)\right. \\
& -\frac{e^{-r(T-t)}}{\theta \sqrt{T-t}} N^{\prime}\left(d_{1, t}\right)\left(\frac{\theta_{L}}{\theta_{H}}\right)^{\frac{1-\gamma}{\gamma}}\left(x_{2}^{*}-B+\frac{k}{\theta_{H}}\right) \\
& \left.+\frac{e^{-r(T-t)}}{\gamma}\left[\left(\frac{k}{\theta_{H}}-B\right) N\left(-d_{1, t}\right)+\left(\frac{k}{\theta_{L}}-\alpha B\right)\left(N\left(d_{1, t}\right)-N\left(d_{3, t}\right)\right)\right]\right\}
\end{aligned}
$$

B. If $x_{1}^{*}=0$ then

$$
X_{t}^{*}=e^{-r(T-t)}\left[\left(x_{2}^{*}-B+\frac{k}{\theta_{H}}\right) N\left(-d_{2, t}\right) \frac{N^{\prime}\left(d_{1, t}\right)}{N^{\prime}\left(d_{2, t}\right)}-\left(\frac{k}{\theta_{H}}-B\right) N\left(-d_{1, t}\right)\right]
$$

and

$$
\pi_{t}^{*}=\frac{\mu-r}{\sigma^{2}}\left[\frac{X_{t}^{*}}{\gamma}+e^{-r(T-t)}\left(\frac{1}{\gamma}\left(\frac{k}{\theta_{H}}-B\right) N\left(-d_{1, t}\right)+\frac{x_{2}^{*}}{\theta \sqrt{T-t}} N^{\prime}\left(d_{1, t}\right)\right)\right]
$$

C. If $x_{1}^{*}=\alpha B$ then

$$
\begin{aligned}
& X_{t}^{*}=e^{-r(T-t)}\left[\left(x_{2}^{*}-B+\frac{k}{\theta_{H}}\right) N\left(-d_{2, t}\right) \frac{N^{\prime}\left(d_{1, t}\right)}{N^{\prime}\left(d_{2, t}\right)}-\left(\frac{k}{\theta_{H}}-B\right) N\left(-d_{1, t}\right)\right] \\
& +e^{-r(T-t)}\left[\alpha B N\left(d_{1, t}\right)+\frac{k}{\theta_{L}}\left(N\left(d_{6, t}\right) \frac{N^{\prime}\left(d_{5, t}\right)}{N^{\prime}\left(d_{6, t}\right)}-N\left(d_{5, t}\right)\right)\right] \\
& -e^{-r(T-t)} \frac{k-\theta_{L} \alpha B}{\theta_{L}}\left(N\left(d_{4, t}\right) \frac{N^{\prime}\left(d_{3, t}\right)}{N^{\prime}\left(d_{4, t}\right)}-N\left(d_{3, t}\right)\right)
\end{aligned}
$$

and

$$
\begin{aligned}
& \pi_{t}^{*}=\frac{\mu-r}{\sigma^{2}}\left\{\frac{X_{t}^{*}}{\gamma}+e^{-r(T-t)} \frac{1}{\theta \sqrt{T-t}}\left(x_{2}^{*}-\alpha B\right) N^{\prime}\left(d_{1, t}\right)\right. \\
& +e^{-r(T-t)}\left(\frac{1}{\gamma}\left(\frac{k}{\theta_{H}}-B\right) N\left(-d_{1, t}\right)-\frac{\alpha B}{\gamma} N\left(d_{1, t}\right)\right) \\
& \left.+e^{-r(T-t)}\left(\frac{1}{\gamma} \frac{k}{\theta_{L}} N\left(d_{5, t}\right)-\frac{1}{\gamma}\left(\frac{k}{\theta_{L}}-\alpha B\right) N\left(d_{3, t}\right)\right)\right\}
\end{aligned}
$$

where $d_{1, t}, d_{2, t}, d_{3, t}, d_{4, t}, d_{5, t}$ and $d_{6, t}$ are defined respectively in (21), (22), (23), (24), (25) and (26).

\section{Illustrative Results and Numerical Examples}

In this section we examine the manager's optimal dynamic trading strategies in both case whether the underperformance penalty is present or not. We study how a change in the underperformance coeffecient or in the penalization boundary coefficient affects the managerial risk taking. Moreover, we analyze the behavior of the optimal trading strategies as the terminal time $T$ is approached.

The expression of $X_{t}^{*}$ and $\pi_{t}^{*}$ are presented in Proposition 3.2 as a function of the state price density $\xi_{t}$. Here we 
analyze the ratio $\frac{\pi_{t}^{*}}{X_{t}^{*}}$, that is the optimal proportion of portfolio value invested in the risky asset. It is possible to show that as $\xi_{t} \rightarrow 0, X_{t}^{*} \rightarrow \infty$ and

$$
\frac{\pi_{t}^{*}}{X_{t}^{*}} \rightarrow \frac{\mu-r}{\gamma \sigma^{2}}:=M
$$

regardless of the values of other parameters, where the constant $M$ is the optimal proportion allocated to the risky asset in the Merton's framework (this result follows along the same lines of Propostion 1 (i) in Carpenter (2000)). In other words, the manager acts as if he were the owner of the fund. On the other hand, as $\xi_{t} \rightarrow \infty, X_{t}^{*} \rightarrow 0$ and $\frac{\pi_{t}^{*}}{X_{t}^{*}} \rightarrow \infty$.

Table 1. Standards parameters

\begin{tabular}{lcl}
\hline Terminal time & $T$ & 2 \\
Evaluation time & $t$ & 1.7 \\
Risk aversion coefficient & $\gamma$ & 0.5 \\
Base fee & $k$ & 0.06 \\
Benchmark & $B$ & 1 \\
Overperformance coefficient & $\theta_{H}$ & 0.3 \\
Underperformance coefficient & $\theta_{L}$ & 0.2 \\
Penalization boundary coefficient & $\alpha$ & 0.25 \\
Initial asset value & $x$ & 1 \\
\hline
\end{tabular}

In the following we refer to a standard set of parameters as displayed in Table 1.

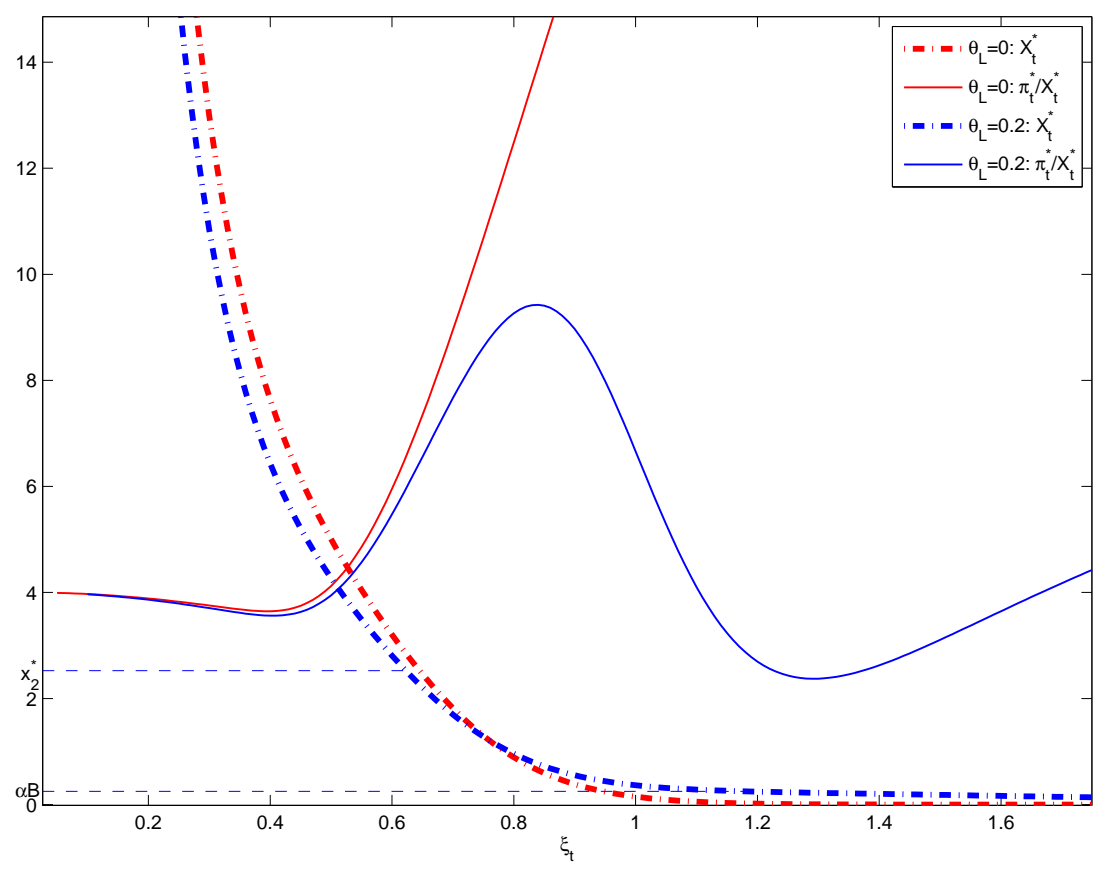

Figure 3. The case $\theta_{L}=0$ and $\theta_{L}=0.2$ 
Figure 3 displays the optimal value of asset portolio $X_{t}^{*}$ (dashed lines) and the optimal proportion ratio $\frac{\pi_{t}^{*}}{X_{t}^{*}}$ (solid line) as a function of the state price density $\xi_{t}$. In particular, we compare the case $\theta_{L}=0$ (absence of underperformance penalty) with the case $\theta_{L}=0.2$. The behavior of $X_{t}^{*}$ in the two cases is similar: $X_{t}^{*}$ is a monotone decreasing function of $\xi_{t}$ and $X_{t}^{*}$ tends to zero as $\xi_{t}$ goes to infinity. Let us now analyze the behavior of $\frac{\pi_{t}^{*}}{X_{t}^{*}}$ Both in cases $\theta_{L}=0$ and $\theta_{L}=0.2$, if the value of $X_{t}^{*}$ is well above $x_{2}^{*}$, there are some stases of the world where $\frac{\pi_{t}^{*}}{X_{t}^{*}} \leq M$ (that we label region of prudence, see also Hodder and Jackwerth (2007)), i.e. the manager chooses a lower asset volatility that he would if he were the owner of the fund (see also Carpenter (2000)).

After that, the behavior of $\frac{\pi_{t}^{*}}{X_{t}^{*}}$ is sensible different in the two cases. In case $\theta_{L}=0$, as $X_{t}^{*}$ increases, $\frac{\pi_{t}^{*}}{X_{t}^{*}}$ converges rapidly to infinity, that is the manager bears a great risk to bring the asset value in the overperformance region. In case $\theta_{L}=0.2$, there is a region (that we label risk seeking region), that occurs few percents below $x_{1}^{*}=\alpha B=$ 0.25 and above $x_{2}^{*}=2.52$, where the optimal proportion $\frac{\pi_{t}^{*}}{x_{t}^{*}}$ is higher than $M$ but not in the undiscriminate manner as in the case $\theta_{L}=0$. For values of $X_{t}^{*}$ well below $\alpha B$, there is a second region of prudence where $\frac{\pi_{t}^{*}}{X_{t}^{*}}<M$. In this region the manager is penalized because the asset value is in the underperformance region. Since $X_{t}^{*}$ is not close to the boundary $\alpha B$, the manager invests with caution in order to limit the loss. In case $\xi_{t} \rightarrow \infty, X_{t}^{*}$ goes to zero and $\pi_{t}^{*}$ approaches zero as well but slowly than $X_{t}^{*}$, then $\frac{\pi_{t}^{*}}{X_{t}^{*}}$ tends to infinity. Notice that in case $\theta_{L}=0, \frac{\pi_{t}^{*}}{X_{t}^{*}}$ tends to infinity at a rate sensible faster than in the case $\theta_{L}=0.2$.

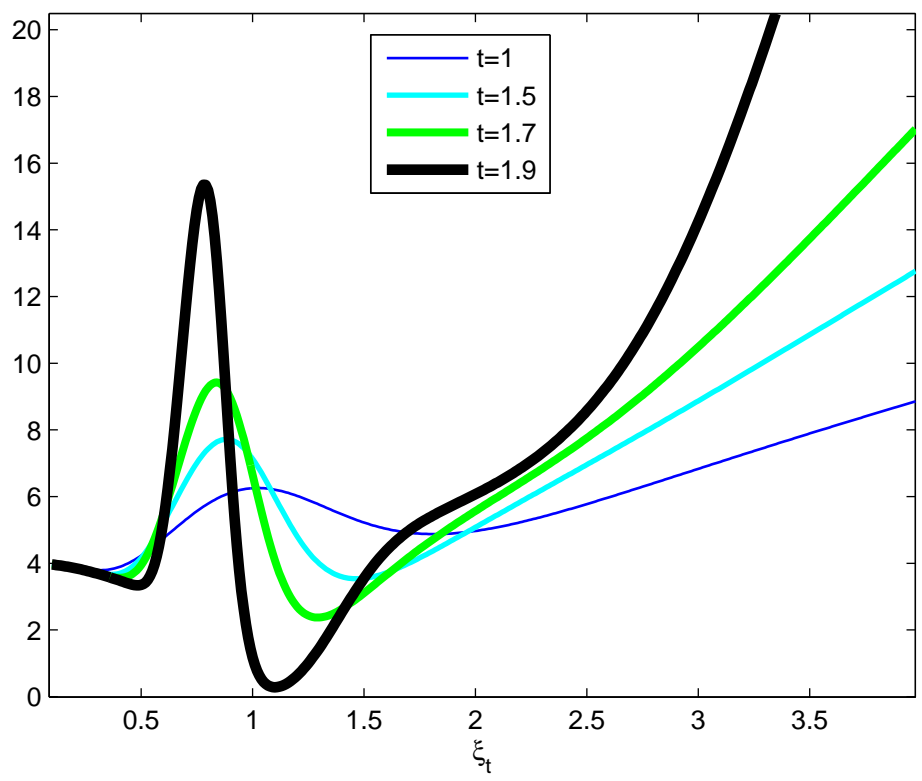

Figure 4. Optimal proportional portfolio at different times

Figure 4 shows the optimal proportion allocated to the risky asset for values of $t \in\{1,1.5,1.7,1.9\}$, the other parameters being the same. Notice the more the evaluation time $t$ is close to the terminal time $T$, the more pronounced are the region of prudence and the risk seeking region. Just prior to $T$, the manager increases the 
riskiness of investments if the asset value is close to the overperformance region. On the other hand, if just prior to $T$ the asset value in one of the two regions of prudence, then there is a little chance to alter significantly the manager's compensation, thus he chooses conservative trading strategies.

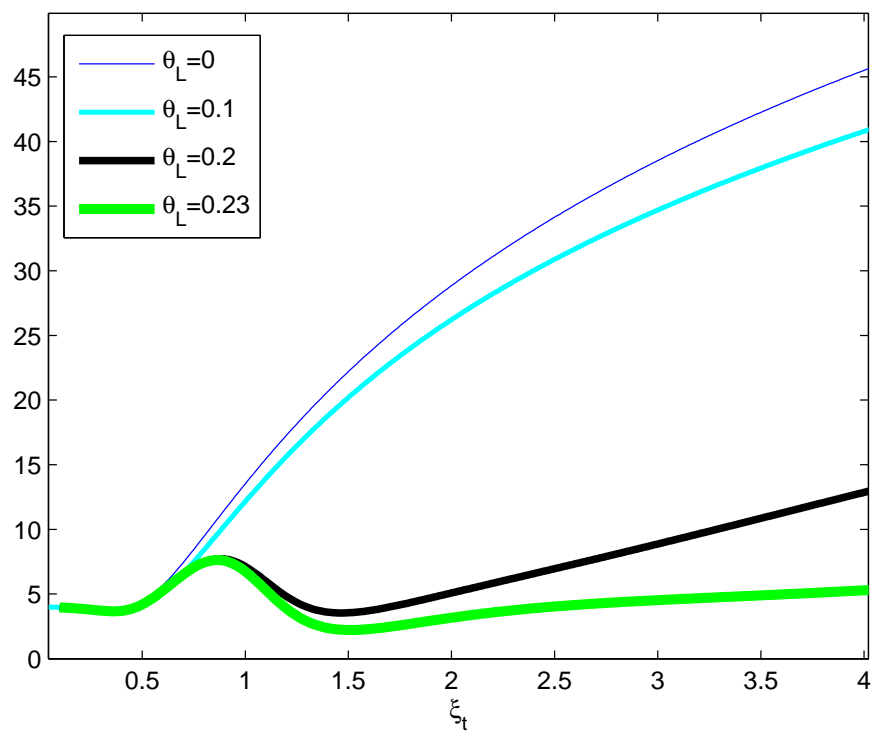

Figure 5. Sensitivity of the optimal proportional portfolio respect to $\theta_{L}$

In Figure 5 it is analyzed the sensitivity of the optimal proportional portfolio respect to the underperformance coefficient $\theta_{L}$. In case $\theta_{L}=0$, after a region of prudence for low values of $\xi_{t}, \frac{\pi_{t}^{*}}{x_{t}^{*}}$ tends rapidly to infinity as $\xi_{t}$ increases. As $\theta_{L}$ increases, the optimal proportional portfolio exhibits risk seeking region and the second region of prudence. Moreover, the greater $\theta_{L}$ is, the less risky are the manager's trading strategies.

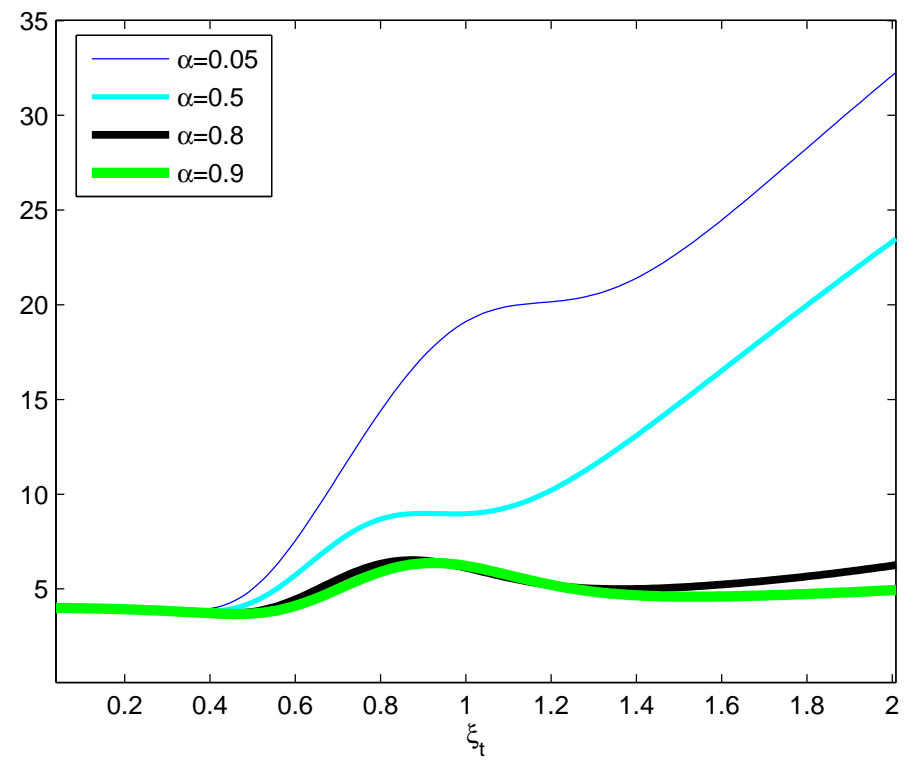

Figure 6. Sensitivity of the optimal proportional portfolio respect to $\alpha$

Finally, Figure 6 examines how a change in the penalization boundary coefficient $\alpha$ impacts on the optimal trading strategies. The more $\alpha$ is close to 1 , the larger is the underperformance region, and then the more prudent are the 
manager's investment trading strategies.

\section{Conclusions}

This research considers a reward scheme for an asset manager that includes both overperformance bonus and underperformance penalty. The analytical solutions of the manager's optimal control problem are presented and the results are illustrated by means of numerical examples. It is shown that the introduction of underperformance penalty may significantly alter the managerial risk taking. In particular, the optimal proportional portfolio shows the presence of a second region of prudence, beyond the one that is also present in case of absence of underperformance penalty, where the manager chooses a lower asset volatility that he would choose if he were the owner of the fund. Moreover, there is a region that we label risk seeking region, where the manager bears a risk greater than if he were the owner of the fund, but not in the undiscriminate manner as in case of absence of underperformance penalty. Finally, a sensitivity analysis is performed respect to the underperformance coefficient as well as the penalization boundary coefficient. Further research should be done to investigate a compensation contract with stochastic benchmark. It should be also of interest to introduce in the compensation scheme caps on both over- and under-performance. Moreover, it should be considered the presence of investment constraints.

\section{References}

Buraschi, A., Kosowski, R., \& Sritrakul, W. (2014). Incentives and endogenous tisk taking: a structural view on hedge fund alphas. The Journal of Finance, 69, 2819-2870. http://dx.doi.org/10.1111/jofi.12167

Carpenter, J.N. (2000). Does option compensation increase managerial risk taking?. The Journal of Finance, 55, 2311-2332. http://dx.doi.org/10.1111/0022-1082.00288

Cox, J.C., \& Huang, C.F. (1989). Optimal consumption and portfolio policies when asset prices follow a diffusion process. Journal of Economic Theory, 49, 33-83. http://dx.doi.org/10.1016/0022-0531(89)90067-7

Cuoco, D., \& Kaniel, R. (2011). Equilibrium prices in the presence of delegated portfolio management. Journal of Financial economics, 101, 264-296. http://dx.doi.org/10.1016/j.jfineco.2011.02.012

Gupta, P., \& Skallsjo, S. (2007). Impact of Manager Compensation on Portfolio Risk and Return. Retrieved from SSRN: http://ssrn.com/abstract $=1021175$

Hodder, J., \& Jackwerth, J. (2007). Incentive contract and hedge fund management. Journal of Financial and Quantitative Analysis, 42, 811-826. http://dx.doi.org/10.1017/S0022109000003409

Karatzas, I., Lehoczky, J., \& Shreve, S.E. (1987). Optimal portfolio and consumption decision for a 'small investor' on a finite horizon. Siam Journal of Control and Optimization, 27, 1157-1186.

Pliska, S.R. (1986). A stochastic calculus model of continuous trading. Mathematics of Operation Research, 11, 371-382. http://dx.doi.org/10.1287/moor.11.2.371

\section{Appendix A: Concavification}

Under the Assumption of Lemma 3.1 the manager's objective function $u(x)$ (see (5)) is not globally concave. Here we discuss the concavification of $u$, that is how to construct the smallest concave function that dominates $u$. The construction of the concavified function $v$ is illustrated in Figure (2).

If $\theta_{H}=\theta_{L}$ and $\alpha<1$, then $u^{\prime}(\alpha B)=u^{\prime}(B)$. Since $u^{\prime}(x)>u^{\prime}(\alpha B)$ for $x \in(0, \alpha B)$ and $u^{\prime}(x)<u^{\prime}(B)$ for $x>B$, there exists a point $x_{2}^{*}$ at which the chord from the point $(\alpha B, u(\alpha B))$ is tangent to $u$ (see Figure (2) panel C).

If $\theta_{H}=\theta_{L}>0$, direct computation shows that $x_{1}$ and $x_{2}$ solutions of (8) are given by

$$
\begin{aligned}
& x_{1}=\left(\frac{1}{\gamma\left(\theta_{H}^{1-\frac{1}{\gamma}}-\theta_{L}^{1-\frac{1}{\gamma}}\right)}\left[\frac{\gamma \theta_{L}^{1-\frac{1}{\gamma}}-\theta_{H}^{1-\frac{1}{\gamma}}}{\theta_{L}}\left(k-\theta_{L} \alpha B\right)+\frac{1-\gamma}{\theta_{H}^{\frac{1}{\gamma}}}\left(k-\theta_{H} B\right)\right]\right)^{+} \\
& x_{2}=x_{1}+\frac{k}{\gamma} \frac{\theta_{H}-\theta_{L}}{\theta_{H} \theta_{L}}+\frac{B}{\gamma}(1-\alpha)
\end{aligned}
$$

One the following three cases happen:

A. $0 \leq x_{1} \leq \alpha B$ 

B. $x_{I}<0$

C. $x_{1>} \alpha B$

In case A., $x_{1}^{*}=x_{1}$ and $x_{2}^{*}=x_{2}$ defined in (16) and (17) respectively are such that the chord from the points $\left(x_{1}^{*}, u\left(x_{1}^{*}\right)\right)$ and $\left(x_{2}^{*}, u\left(x_{2}^{*}\right)\right)$ is tangent to $u$ at $x_{1}^{*}$ and $x_{2}^{*}$ (see Figure 2, panel A). In case B. set $\mathrm{x}_{1}^{*}=0$, whereas in case C. set $x_{1}^{*}=\alpha B$. In both cases, $x_{2}^{*}$ is the point at which the chord from the point $\left(x_{1}^{*}, u\left(x_{1}^{*}\right)\right)$ is tangent to $u$ (see Figure 2, panels B and C respectively).

Finally, notice that $v(x)=u(x)$ for $x \in\left[0, x_{1}^{*}\right) \cup\left(x_{2}^{*},+\infty\right)$ and $v$ is linear otherwise, then $v$ is the smallest concave function such that $v(x) \geq u(x)$ on $(0,+\infty)$.

\section{Appendix B: Proof of Proposition 3.2}

Consider the concavified optimization probem

$$
\left.\max _{x_{T}} \mathbf{E}\left[u\left(X_{T}\right)\right)\right]
$$

with $v$ defined in (9), subject to $\mathbf{E}\left[\xi_{T} X_{T}\right] \leq x$ and $X_{T} \geq 0$. From the study of maximization of expected utility of terminal wealth (see Cox and Huang (1989), Karatzas (1987) and Pliska (1986)), the optimal random terminal asset value is $X_{T}^{*}=i\left(\lambda^{*} \xi_{T}\right)$, where

$$
i(y)=\left\{x \in[0, \infty): v^{\prime}(x)=y\right\}
$$

is the inverse marginal utility of the concavified utility $v$ and $\lambda^{*}$ solves $\mathbf{E}\left[\xi_{T} i\left(\lambda^{*} \xi_{T}\right)\right]=x$. Thus the optimal terminal wealth is

$$
\begin{gathered}
X_{T}^{*}=\frac{1}{\theta_{H}}\left(\left(\frac{\lambda \xi_{T}}{\theta_{H}}\right)^{-\frac{1}{\gamma}}-k+\theta_{H} B\right) \mathbb{I}_{\left\{\lambda \xi_{T} \leq v^{\prime}\left(x_{2}^{*}\right)\right\}}+\alpha B \mathbb{I}_{\left\{v^{\prime}\left(x_{2}^{*}\right)<\lambda \xi_{T}<v^{\prime}\left(x_{1}^{*}\right)\right\}}+ \\
+\frac{1}{\theta_{L}}\left(\left(\frac{\lambda \xi_{T}}{\theta_{L}}\right)^{-\frac{1}{\gamma}}-k+\alpha \theta_{L} B\right) \mathbb{I}_{\left\{v^{\prime}\left(x_{1}^{*}\right)<\lambda \xi_{T}<v^{\prime}(0)\right\}}
\end{gathered}
$$

Consider the terminal wealth defined in (18) and let $Y$ be another feasible payoff that is not almost surely equal to $X_{T}^{*}$. Then

$$
\begin{aligned}
\mathbf{E}\left[u(Y)-u\left(X_{T}^{*}\right)\right] & =\mathbf{E}\left[u(Y)-v\left(X_{T}^{*}\right)\right] \\
& \leq \mathbf{E}\left[v(Y)-v\left(X_{T}^{*}\right)\right] \\
& \leq \mathbf{E}\left[v^{\prime}\left(X_{T}^{*}\right)\left(Y-X_{T}^{*}\right)\right] \\
& \leq \mathbf{E}\left[\lambda \xi_{T}\left(Y-X_{T}^{*}\right)\right] \\
& \leq \lambda\left(\mathbf{E}\left[\xi_{T} Y\right]-x\right) \\
& \leq 0
\end{aligned}
$$

where (19b) follows from the fact that $v(x) \geq u(x),(19 \mathrm{c})$ follows from the concavity of $v$, (19d) follows from the definition of $X_{T}^{*}$ as the inverse of the marginal utility of $v$. Note that the optimal terminal wealth for the manager's problem never takes on values between $x_{1}^{*}$ and $x_{2}^{*}$.

Furthermore, the optimal assets value at time $t$ is given by

$$
X_{t}^{*}=\mathbf{E}_{t}\left[\frac{\xi_{T}}{\xi_{t}} X_{T}^{*}\right]
$$

From the definition of state price deflator (7), we get for $a, b>0$ and $\alpha>0$ 


$$
\boldsymbol{E}_{t}\left[\xi_{T}^{\alpha} \mathbb{I}_{\left\{a<\xi_{T}<b\right\}}\right]=\xi_{t}^{\alpha} e^{-\alpha\left(r+\frac{\theta^{2}}{2}\right)(T-t)+\frac{\alpha^{2}}{2} \theta^{2}(T-t)}[N(d+\alpha \theta \sqrt{T-t})-N(c+\alpha \theta \sqrt{T-t})]
$$

where

$$
\mathrm{c}=\frac{\log \left(\frac{\xi_{t}}{b}\right)-\left(r+\frac{\theta^{2}}{2}\right)(T-t)}{\sqrt{T-t}} \text { and } d=\frac{\log \left(\frac{\xi_{t}}{a}\right)-\left(r+\frac{\theta^{2}}{2}\right)(T-t)}{\sqrt{T-t}}
$$

By using (20), it is possible to derive the explicit form of $X_{t}^{*}$ in (10), (12) and (14), where

$$
\begin{aligned}
& d_{1, t}= \frac{\log \left(\frac{\lambda^{*} \xi_{t}}{v^{\prime}\left(x_{2}^{*}\right)}\right)-\left(r-\frac{\theta^{2}}{2}\right)(T-t)}{\theta \sqrt{T-t}} \\
& d_{3, t}= \frac{d_{2, t}=d_{1, t}-\frac{\theta \sqrt{T-t}}{\gamma}}{\theta \sqrt{T-t}} \\
& d_{5, t}=\left.\frac{\lambda^{*} \xi_{t}}{u^{\prime}(0)}\right)-\left(r-\frac{\theta^{2}}{2}\right)(T-t) \\
& d_{4, t}=d_{3, t}-\frac{\theta \sqrt{T-t}}{\gamma} \\
& d_{6, t}=d_{5, t}-\frac{\theta \sqrt{T-t}}{\gamma}
\end{aligned}
$$

Finally, the optimal trading strategy that generates the optimal level of wealth $X_{t}^{*}$ may be calculated as follows

$$
\pi_{t}^{*}=-\frac{\mu-r}{\sigma^{2}} \xi_{t} \frac{\partial X_{t}^{*}}{\partial \xi}
$$

and after some algebra (11), (13) and (15) follow.

\section{Notes}

Note 1. Notice that if $\theta_{L}=0$ the compensation is the same of Carpenter (2000), whereas if $\alpha=1$ the compensation has the same form of Cuoco and Kaniel (2011).

Note 2. The fact that the manager has infinite marginal utility at zero wealth implies that the optimal investment strategy guarantees positive fee at time $T$. 